\title{
Fourth International Workshop on Verification and Evaluation of Computer and Communication Systems (VECoS 2010)
}

\author{
Paris, France \\ 1-2 July 2010
}

Editors:

Kamel Barkaoui

Bruno Monsuez 


\section{Abstract}

The International Workshop on Verification and Evaluation of Computer and Communication Systems (VECoS) was created by an Euro-Maghrebian network of researchers in computer science. VECoS 2010 is co-organised by the Conservatoire National des Arts et Métiers and École Nationale Supérieure de Techniques Avancées with cooperation of Méthodes Formelles pour les Systèmes Logiciels et Matériels Group, and sponsored by Formal Methods Europe.

This conference is sponsored by: Formal Methods Europe 


\section{Full Synopsis}

The International Workshop on Verification and Evaluation of Computer and Communication Systems (VECoS) was created by an Euro-Maghrebian network of researchers in computer science. VECoS 2010 is co-organised by the Conservatoire National des Arts et Métiers and École Nationale Supérieure de $\underline{\text { Techniques Avancées with cooperation of Méthodes Formelles pour les Systèmes Logiciels et Matériels }}$ Group, and sponsored by Formal Methods Europe.

The first edition tooks place in Algiers on 5-6 May 2007 (VECoS 2007), the second edition in Leeds on 23 July 2008 (VECoS 2008), the third edition in Rabat on 2-3 July 2009 (VECoS 2009).

The aim of VECoS workshop is to bring together researchers and practitioners in the areas of verification, control, performance, quality of service, dependability evaluation and assessment, to discuss the state of the art for solving the challenges facing us today in various modern computer and communication systems in which functional and extra functional properties are strongly interrelated. Thus, the main motivation for VECOS is to encourage the cross-fertilisation between formal verification and evaluation approaches, methods and techniques, especially those based on the specification formalisms for concurrent, distributed and soft/hard systems. Beyond its technical and scientific goals, another main purpose of VECoS is to promote collaboration between participants in research and education in the area of computer science and engineering. 


\section{Editors}

This conference was edited by:

Kamel Barkaoui, CEDRIC - CNAM, France (Chair)

Bruno Monsuez, ENSTA - LEI, France (Co-chair)

\section{Program Co-chairs}

Denis Poitrenaud LIP6 - University Paris 6, France

Loïc Corenson LIST-CEA, France

\section{Steering Committee}

Hassane Alla LAG-INPG Grenoble, France

Nadjib Badache LSI-USTHB Algiers, Algeria

Kamel Barkaoui CEDRIC-CNAM Paris, France (Chair)

Rahma Ben Ayed SysCom-ENIT Tunis, Tunisia

Karim Djemame University of Leeds, UK

Karim Djouani Université Paris Est LISSI, France

Said El Hajji University of Mohammed V - Agdal, Rabat, Morocco

Mohamed Kaaniche LAAS CNRS Toulouse, France

Bruno Monsuez ENSTA - LEI, Paris, France

Patrice Moreaux LISTIC University of Savoie Annecy, France

\section{Programme Committee}


Hassane Alla, Univ Grenoble, France

Nasreddine Aoumeur, Univ Magdeburg, Germany

Djamil Aissani, LAMOS Bejaia, Algeria

Yamine Ait-Ameur, LISL ENSMA Poitiers, France

Karim Baïna, ENSIAS Rabat, Morocco

Kamel Barkaoui, Cedric-CNAM Paris, France

Faïza Belala, Univ. Constantine, Algeria

Nejib Ben Hadj-Alouane, ENIT Tunis, Tunisia

Narjes Ben Rajeb, INSAT Tunis, Tunisia

Saddek Bensalem, VERIMAG Grenoble, France

Hanifa Boucheneb, VeriForm, École Polytechnique de Montréal, Canada

Mario Bravetti, Univ. Bologna, Italy

Boutheina Chetali, Gemalto, France

Loïc Corenson, LIST-CEA, France, (Co-Chair)

Jean-Michel Couvreur, LIFO Univ. Orlés, France

Isabel Demongodin, LSIS Marseille, France

Maria Pia Fanti, Politecnico di Bari, Italy

Mamoun Filali-Amine, IRIT Toulouse, France

Francesco Flammini, Universitài Napoli , Italy

Serge Haddad, LSV ENS Cachan, France

Malika Ioulalen, USTHB Alger, Algeria

Jorge Julvez, University of Zaragoza., Spain

Mohamed Khalgui, Martin Luther University, Germany

Hanna Klaudel, IBISC-CNRS Univ. Evry, France

Ouajdi Korbaa, LI3, ISITCom, Tunisia

Fabrice Kordon, LIP6 Univ. Paris 6, France

Zhiwu Li, Xidian University, China

Mourad Maouche, Philadephia University, Amman, Jordan 
Abdelhamid Mellouk, LISSI Univ Paris 12, France

Bruno Monsuez, ENSTA Paris, France

Hassan Mountassir, LIFC University France-Comté, France

Joel Ouaknine, Oxford University, UK

Nihal Pekergin, LACL, University Paris Est, France

Jean-François Petin, CRAN Nancy, France

Laure Petrucci, LIPN Univ. Paris Nord, France

Denis Poitrenaud, LIP6 Univ. Paris 6, France, (Co-Chair)

Jean-François Raskin, Université Libre de Bruxelles (ULB), Belgium

Riadh Robbana, LIP2/EPT Carthage, Tunisia

Jean-Marc Roussel, LURPA ENS Cacahn, France

Olivier-H Roux, IRCCYN Nantes, France

Brigitte Rozoy, LRI - CNRS Univ. Paris Sud, France

Natalia Sidorova, Technische universiteit Eindhoven, Nederland

Grégoire Sutre, LaBRI Bordeaux, France

Thouraya Tebibel, ESI, Algeria

Tayssir Touili, LIAFA Univ Paris 7, France

François Vernadat, LAAS-CNRS Toulouse, France

Anrong Wang, Xidian University, China

Karsten Wolf, Universitaet Rostock, Institut fuer Informatik, Germany

Habib Youssef, ISITCH Sousse, Tunisia 


\section{Papers:}

Raymond Devillers Deadlock Avoidance, Non-Linearity and Games http://dx.doi.org/10.14236/ewic/VECOS2010.1

Laurent Fribourg Simulation + Uncertainty $=$ Model Checking http://dx.doi.org/10.14236/ewic/VECOS2010.2

Hans-Michael Hanisch Building Closed-loop Models for Discrete Controller Design http://dx.doi.org/10.14236/ewic/VECOS2010.3

Mohammed-Amine Belhaj Seboui, Nejib Ben Hadj-Alouane, Gwenael Delaval, Eric Rutten and Moez Yeddes A Decentralized Supervisory Control Approach for Distributed Adaptive Systems http://dx.doi.org/10.14236/ewic/VECOS2010.4

Annie Choquet-Geniet, Galle Skapin-Largeteau and Abdoulaye Ouattara Integrating PFairness within a Model Based Scheduling Tool http://dx.doi.org/10.14236/ewic/VECOS2010.5

Chafia Bouanaka, Faiza Belala and Kamel Barkaoui A Tile Logic Based Semantics for Mobile Software Architectures http://dx.doi.org/10.14236/ewic/VECOS2010.6

Mohammed Amin Ben Amar, Hervé Camus and Ouajdi Korbaa Formalization of Cyclic Scheduling Problems with Assembly Tasks and Work-in-Progress Minimization http://dx.doi.org/10.14236/ewic/VECOS2010.7

Laïd Kadloul, Karim Djouani and Walid Tfaili Using Timed Colored Petri Nets and CPN-tool to Model and Verify TRBAC Security Policies http://dx.doi.org/10.14236/ewic/VECOS2010.8

Brahim Nasraoui, Syrine Ayadi and Riadh Robbana SBMC: Symmetric Bounded Model Checking http://dx.doi.org/10.14236/ewic/VECOS2010.9

Hamid Reza Mahrooghi, Mohammad Hashem Haghighat and Rasool Jalili

Formal Verification of Authentication-Type Properties of an Electronic Voting Protocol Using mCRL2 http://dx.doi.org/10.14236/ewic/VECOS2010.10

Hamdi Eltaief and Habib Youssef $\quad$ AMLCC: Adaptive Multi-Layer Connected Chains Mechanism for Multicast Sender Authentication of Media-Streaming http://dx.doi.org/10.14236/ewic/VECOS2010.11

Diana El Rabih, Gaël Gorgo, Nihal Pekergin and Jean-Marc Vincent Steady-State Property Verification: a Comparison Study $\quad$ http://dx.doi.org/10.14236/ewic/VECOS2010.12 